\begin{tabular}{|l|l|r|}
\hline & COMPARATIVE ANALYSIS AND EVALUATION OF THE APPLICATION OF & \\
& DEEP LEARNING TECHNIQUES TO CYBERSECURITY DATASETS & \\
Ingeniería e Industria & & BUILDING \\
\hline RESEARCH ARTICLE & Xavier A. Larriva-Novo, Mario Vega-Barbas, Víctor A. Villagrá, Julio Berrocal & TECHNOLOGY \\
\hline
\end{tabular}

\title{
COMPARATIVE ANALYSIS AND EVALUATION OF THE APPLICATION OF DEEP LEARNING TECHNIQUES TO CYBERSECURITY DATASETS
}

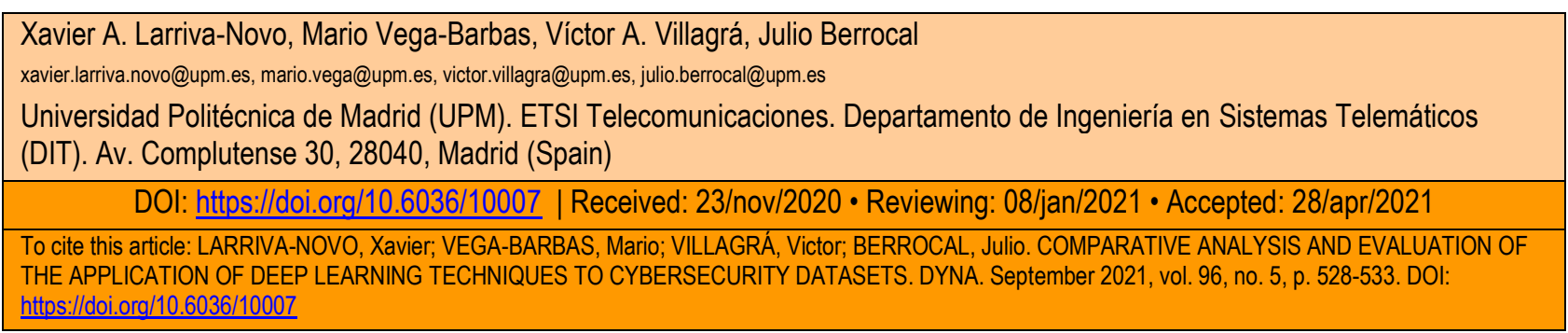

\section{ABSTRACT:}

Cybersecurity has been highlighted in recent years to protect information systems. Various methods, techniques, and tools have been used to make the most of the existing vulnerabilities of these systems. Therefore, it is essential to develop and improve new technologies, as well as intrusion detection systems to detect possible threats. However, the use of these technologies requires highly qualified cybersecurity personnel to analyze the results and improve the accuracy of the results. Therefore, this generates the need to research and develop new cybersecurity systems with high performance to efficiently analyze and resolve such results. This research presents the application of machine learning techniques for real traffic classification, intending to identify possible attacks. The study has been carried out using machine learning tools applying deep learning algorithms such as multilayer perceptron and long short term memory. Additionally, this paper presents a comparison between the results obtained by the application of the algorithms and non-deep learning algorithms such as random forest and decision tree. Finally, the results obtained show that the long short-term memory algorithm is the one that provides the best results in terms of accuracy and logarithmic loss.

Key Words: Artificial Intelligence, LSTM, neural networks, intrusion detection system, UGR16.

\section{INTRODUCTION}

Artificial Intelligence (AI) has been extensively studied over the last few years for its application in the field of cybersecurity. Specifically, machine learning techniques have been used to create new algorithms or refine existing ones in this field, to obtain more robust systems in the face of more sophisticated threats.

One of the most common applications where machine learning techniques have had the strongest impact is intrusion detection systems (IDSs) [1], [2]. These detection systems are divided into two classes, IDSs based on signatures and IDSs based on anomaly detection. IDSs based on signature detection use different rules that allow the identification of a possible attack at the host or network level. Generally, these rules require the knowledge of a cybersecurity expert to perform the correct detection of possible attacks.

On the other hand, IDSs based on anomaly detection allows differentiating between traffic and suspicious behavior. Although these types of IDSs represent robust detection solutions, they must deal with a major problem that impairs their efficiency, i.e., false-positive detection [3]. Due to the number of false positives generated by these IDSs, human capital experts in cybersecurity must analyze and investigate the veracity of the results obtained [4].

These problems have become research and development challenges as the potential for new attacks has increased significantly with the use of more sophisticated techniques. For this reason, the application and use of machine learning techniques in the development of new IDSs have acquired considerable importance. These Al techniques offer the possibility of automating the process of analyzing data traffic managed by telematics systems accurately and efficiently.

The main objective of this research is to analyze different machine learning methods for the classification of real traffic and the identification of possible attacks. Specifically, this paper proposes a comparative study between traditional machine learning techniques and the use of deep learning algorithms such as Long Short Term Memory (LSTM) [5]and Multi-Layer Perceptron (MLP) [6]. The choice to use MLP is mainly since the feature dimensionality is significantly reduced after selection. Therefore, an MLP with all layers fully connected can make sufficient use of all remaining features to extract hidden information with only a few parameters, thus achieving good performance without expensive computation. In the case of the LSTM network, it was selected because of its ability to store both long-term and short-term information since cybersecurity attacks have specific behaviors in certain time windows.

\begin{tabular}{|c|c|c|}
\hline & Publicaciones DYNA SL - c) Mazarredo n69 - 20 -- 48009-BILBAO (SPAIN) \\
Tel +34 944237566 - www.revistadyna.com - email: dyna@revistadyna.com
\end{tabular}




\begin{tabular}{|c|l|r|}
\hline & COMPARATIVE ANALYSIS AND EVALUATION OF THE APPLICATION OF & \\
Ingeniería e Industria & & \\
& & BUILDING \\
\hline RESEARCH ARTICLE & Xavier A. Larriva-Novo, Mario Vega-Barbas, Víctor A. Villagrá, Julio Berrocal & TECHNOLOGY \\
\hline
\end{tabular}

With the use of the LSTM network, we intend to evaluate this memory feature it offers despite the computational cost it exerts on training.

The results of this analysis have focused on determining which solution provides the best relationship between the accuracy of the results and their logarithmic loss (Logloss).

Traditionally, different studies have used the KDDCup 1999 dataset [7]to implement and analyze these developments. However, this dataset is currently considered obsolete due to how it was generated, labeled, and the age of the attacks it covers [8]. Therefore, this paper proposes the use of a new cybersecurity dataset, UGR16, built from real traffic and updated attacks [9].

Thus, Section 2 of this paper presents a state of the art of works related to the training of various intrusion detection systems that apply machine learning algorithms. Sections 3 and 4 detail the problem analyzed by this work and the methodology proposed to address it. Then, Section 5 presents the results obtained using the proposed methodology and a comparison between the aforementioned algorithms and traditional machine learning algorithms such as Random Forest [10]and Decision Tree [11]. Finally, the conclusions of the work performed are explained in section 6 .

\section{RELATED WORKS}

There are three main classification models in the use of machine learning techniques: supervised, unsupervised and semi-supervised training. The supervised training model makes predictions of categorical values based on a set of characteristics or features, generating predictions based on past or present time series. Examples of these algorithms are Logistic Regression, Naïve Bayes, k-nearest neighbors (KNN), Random Forest, or Decision Trees [12]. The unsupervised training model is usually used when there is no expected value in the output of the result. In this case, these models present several proposals for information management. The first proposal consists of a grouping of data into different classes; the second consists of extracting the most significant patterns from the data and grouping them; the third proposal, called dimension reduction, consists of reducing the characteristics or features of the values of the analyzed data set [13].

Deep learning techniques consist of a set of machine learning-type algorithms that act at multiple levels [14]. Several algorithms based on deep learning techniques have been applied to the study of IDS, such as Long short-term memory (LSTM) [5]. This technique offers a component called forget gate that allows learning temporal features or characteristics in long-time sequences [15]. The study described in [16]presents how the LSTM algorithm was applied to the development of an IDS focused on anomaly detection, using the KDD Cup 1999 dataset as a development basis. This study determined the initial hyperparameters by measuring the performance and results obtained with an accuracy of $96.93 \%$. Also, this study applied the concept of the language model to modify and condition the data. Thus, the result obtained was more accurate compared to other algorithms, although the time spent on training the algorithm was the longest [17].

In [18]a study similar to those previously analyzed is presented, obtaining an accuracy of $93.82 \%$. The authors analyze a data conditioning model that consists of the normalization of numerical values in z-score and string values in numerical values with a unique identifier. This normalization allowed to improve the accuracy of the results obtained in comparison with other conventional techniques used in machine learning algorithms. In this line, the study presented in [19][20] used Deep Belief Networks (DBN), and Stacked Auto Encoders type algorithms for the development of an IDS focused on anomaly detection in a $5 \mathrm{G}$ type network for mobile architectures. In this case, a graphics processing unit (GPU) was used to significantly decrease the learning process time.

Multi-layer perceptron (MLP) has been studied in the application of various IDS, as is the case of [21], which obtained an accuracy of 91\% using two hidden layers for its development and training the system with the Defense Advanced Research Projects Agency (DARPA) dataset [22]Other works focused on the study of MLP have focused on addressing the problem of false positives, such as [6], which obtained an accuracy of $95.87 \%$, using the KDDCup 99 dataset.

As has been shown, there are a remarkable number of studies aimed at improving accuracy and decreasing the number of false positives in the final result since a false positive in cybersecurity can decrease confidence in the tools. In general, these studies determine which Recurrent Neural Networks (RNN) type algorithms generate the most reliable results [23]. However, as the authors have pointed out, these studies use the KDDCup 99 dataset [24], which is outdated.

\section{PROBLEM}

This research proposes the application of deep learning machine learning algorithms for the analysis of real network traffic labeled based on current attacks. The application consists of determining the best results by evaluating them in terms of accuracy and logloss. The first is an MLP type algorithm, and the second is an LSTM type algorithm. LSTM represents a recent machine learning architecture based on recurrent neural networks, less explored but offering better performances in recent works [25]. MLP, on the 


\begin{tabular}{|c|r|r|}
\hline & COMPARATIVE ANALYSIS AND EVALUATION OF THE APPLICATION OF & \\
Ingeniería e Industria & & \\
& & BUILDING \\
\hline RESEARCH ARTICLE & Xavier A. Larriva-Novo, Mario Vega-Barbas, Víctor A. Villagrá, Julio Berrocal & TECHNOLOGY \\
\hline
\end{tabular}

contrary, has been addressed by more research works, so its study and application have been greater according to the studies analyzed in Section 2.

Regarding the dataset used in the definition of the analysis models, the studies presented in the previous section generally use the KDDCup 99 dataset, which has become a reference for the various investigations of intrusion detection systems based on machine learning techniques [24]. The main problem of this dataset lies in its currency. Therefore, an updated dataset, the UGR16, is proposed as the basis of analysis. Thus, it is possible to perform training based on data related to real traffic and current attacks to properly identify the computational capacity of the analyzed algorithms. This analysis was evaluated using metrics commonly used in this field, such as accuracy, performance, and Logloss.

\section{PROPOSED METODOLGY}

This research proposes the application of algorithms highlighted in recent literature, of deep learning LSTM and MLP type, for the analysis of real network traffic labeled with current attacks. This analysis has used the UGR16 dataset, introduced in the year 2016, which contains real traffic labeled with updated attacks [9]. The main advantage of this dataset over the previous ones is its usefulness to evaluate IDS since it considers the long-term evolution of network traffic and its periodicity.

Additionally, this work contemplates evaluating the performance of the implemented solutions and obtaining the training times required by the algorithms. These times will depend, in addition to the model and its characteristics, on the underlying technology, so the study should show the results taking into account whether the models are executed on a Central Processing Unit (CPU) or a GPU.

The metrics used to perform the proposed analysis are accuracy and logloss. Accuracy (AC) is considered one of the most important performance indicators and determines the number of correctly predicted records of a class. The True Positive (TP) value is equivalent to the correctly predicted values corresponding to a class, in this case, the classification that determines a background or attack type attack. The False Positive (FP) value is the number of predictions that are not equivalent to the corresponding class. The True Negative (TN) is the result of those values that are presented, corresponding to the number of records that are identified as normal. The False Negative (FN) is the incorrectly predicted result for a corresponding class. Equation (1) shows how the accuracy and the relationship of these parameters are calculated. Table I presents the corresponding confusion matrix.

$$
A C=\frac{T P+T N}{T P+T N+F P+F N}
$$

\begin{tabular}{|c|c|c|c|}
\hline \multirow{2}{*}{} & \multicolumn{2}{|c|}{ Predicate Class } \\
\cline { 2 - 3 } & Attack & Attack & No_attack \\
\hline \multirow{2}{*}{ Current Class } & TP & FN \\
\cline { 2 - 3 } & No_attack & FP & TN \\
\hline
\end{tabular}

Table l: Confusion matrix, actual class/predicted class

Logloss is used in the analysis of machine learning algorithms to show the reliability of the system. The Logloss value increases as the probability of the predicted label differ from the value of the actual label [26]. The objective of a model based on machine learning techniques is to minimize this value.

$$
\text { Logloss }=-\frac{1}{N} \sum_{i=0}^{N} \sum_{j=0}^{M} y_{i, j} \log \left(p_{i, j}\right)
$$

Equation (2) details the calculation of Logloss, where $\mathrm{N}$ is the total observations in the data set; $\mathrm{M}$ is the number of class labels; $y i, j$ is 1 when observation i corresponds to class j; otherwise, it is 0 ; and $p i, j$ is the prediction probability that this observation $i$ belongs to class j [25].

Finally, it should be noted that this research has taken into account the technology on which the proposed deep learning algorithms have been executed to show a deeper analysis. For this purpose, two execution environments were defined. The first one consists of an Intel Core i7-7700HQ 2.80Ghz processor, SSD ROM, $32 \mathrm{~Gb}$ RAM, and 64-bit Windows operating system. The second one consists of a GTX 1050 4GB GPU integrated on the same CPU mentioned above

\begin{tabular}{|c|c|c|}
\hline & Publicaciones DYNA SL - c) Mazarredo n69 - $2^{\circ}$-- 48009-BILBAO (SPAIN) \\
Tel +34 944237566 - www.revistadyna.com - email: dyna@revistadyna.com
\end{tabular}




\begin{tabular}{|c|l|r|}
\hline & COMPARATIVE ANALYSIS AND EVALUATION OF THE APPLICATION OF \\
Ingeniería e Industria & & \\
\hline RESEARCH ARTICLE & Xavier A. Larriva-Novo, Mario Vega-Barbas, Víctor A. Villagrá, Julio Berrocal & BUILDING \\
TECHNOLOGY \\
\hline
\end{tabular}

\section{EXPERIMENTATION AND DISCUSSION OF RESULTS}

The research focused on the first week of the UGR16 dataset (subset) and whose distribution of characteristics is shown in Table II, in which they are shown in terms of percentage as well as their relationship with the original dataset.

\begin{tabular}{|c|c|c|}
\hline & Features March Week 1 & Subset-March Week 1 \\
\hline Size & $45 \mathrm{~GB}$ & $4 \mathrm{~GB}$ \\
\hline Number of labeled flows & $\approx 240 \mathrm{M}$ & $637 \mathrm{M}$ \\
\hline TCP Protocol & $63.6 \%$ & $36.03 \%$ \\
\hline UDP Protocol & $34.4 \%$ & $1.09 \%$ \\
\hline Gre Protocol & $1.8 \%$ & $0.06 \%$ \\
\hline Esp Protocol & $0.1 \%$ & $0.05 \%$ \\
\hline Ipip Protocol & $0.07 \%$ & 0.01 \\
\hline Ipv6 Protocol & $0.01 \%$ & $\approx 0.0 \%$ \\
\hline Cbt Protocol & $\approx 0.0 \%$ & $\approx 0.0 \%$ \\
\hline Background Traffic Data & $\approx 0.0 \%$ & $99.8 \%$ \\
\hline Anomaly-Spam Flow & $99.18 \%$ & $0.12 \%$ \\
\hline Anomaly-Sshscan Flow & $0.47 \%$ & $\approx 0.08 \%$ \\
\hline
\end{tabular}

Table II: Characteristics of the analyzed dataset

The total size of the subset (March Week 1) amounts to 45 GB of information, so it was necessary to adopt management and administration measures for large data sets. Although there are different ways to address this problem, this research opted for the use of a reduced dataset formed from random entries of the initial subset. For the new reduced dataset, a similar relationship was established with the distribution of features presented by the source subset, as detailed in Table II.

Finally, although there are different libraries for the development of deep machine learning models, this research was based on the Keras library [27]with TensorFlow [28]as a back-end. In addition, the works analyzed in Section 2 were used as a basis for determining the number of hidden layers in the implemented MLP architecture and the characteristics of the LSTM-based architecture.

\subsection{Binary Classification}

To establish the MLP configuration, the 13 variables of the dataset (detailed in the supplementary material) have been used, being transformed into 38 features through binary input conversion and one hot encoding_29]. The epoch (number of iterations on the training data) used is 100, with early stooping [28]. In the tests performed, the best accuracy was achieved with 50 hidden nodes and five layers. The accuracy of the predictions was calculated using $20 \%$ of the random values of the reduced subset, while the training was performed with $80 \%$.

To test the performance of LSTM, the 13 features of the UGR16 dataset were encoded into values with unique identifiers using label encoder_[30], as mentioned in the same section, by which after rigorous testing obtained the best accuracy. The neural network structure was configured with three layers; the first one considered the input nodes, the second one implemented a LSTM layer, and the output layer was ordered with two nodes. The best results of this configuration were obtained with three epochs.

This study compares the accuracy score results obtained with other IDS results based on Section 3. The results presented in Figure. 1 show that MLP and LSTM provide better accuracy results.

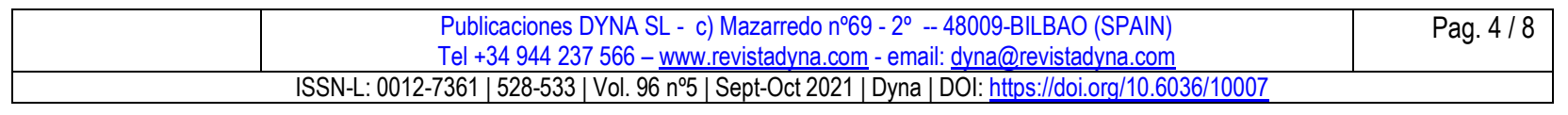




\begin{tabular}{|c|l|r|}
\hline & COMPARATIVE ANALYSIS AND EVALUATION OF THE APPLICATION OF & \\
Ingeniería e Industria & & \\
& & BUILDING \\
\hline RESEARCH ARTICLE & Xavier A. Larriva-Novo, Mario Vega-Barbas, Víctor A. Villagrá, Julio Berrocal & TECHNOLOGY \\
\hline
\end{tabular}

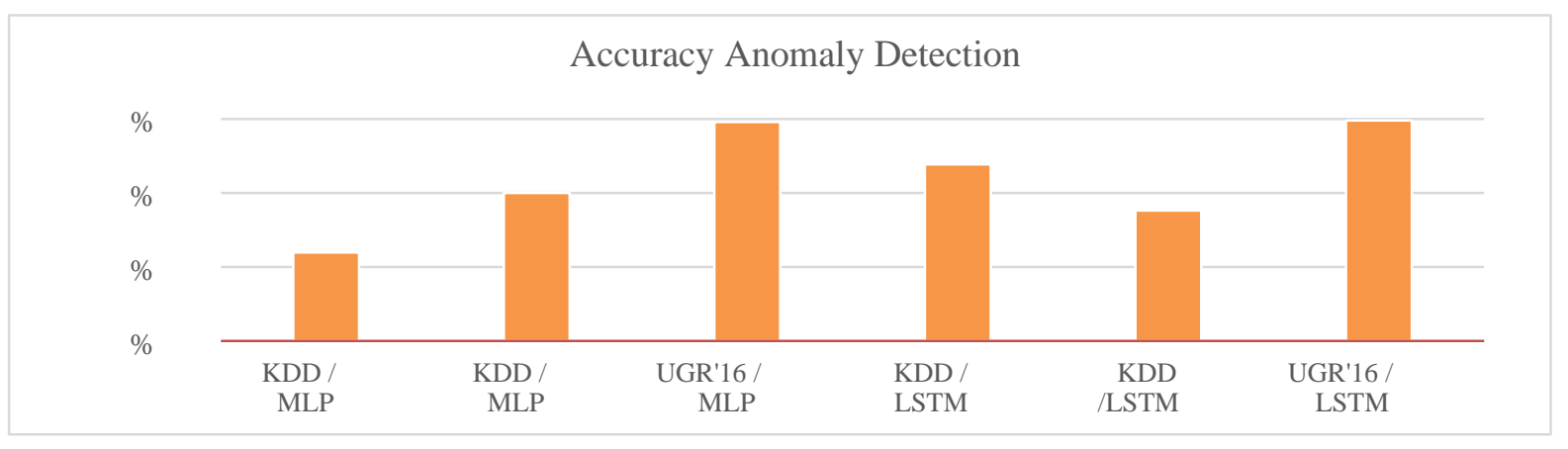

Figure 1 Accuracy in anomaly detection

As a complement to this study, some tests were performed with other non-DNN type algorithms, such as Random Forest (RF) [10], with a random_state of 3 and several estimators equal to 103; and Decision Tree (DT), whose criterion uses "Gini" and as well as the variable "best category" for the search strategy [11][17], [31]. For these tests, the same data set was used for training and prediction like the one used for MLP and LSTM. The results are presented in Table III.

Looking at these results, we can see that, when testing the algorithms with the UGR16, the accuracy presented high values of approximately $99 \%$. However, the Logloss value gave us the knowledge to decide which system has a better performance

\begin{tabular}{|c|c|c|c|c|}
\hline \multirow{2}{*}{} & \multicolumn{2}{|c|}{ Deep Neural Network } & \multicolumn{2}{c|}{ Non-Deep Neural Network } \\
\cline { 2 - 5 } & $\boldsymbol{M L P}$ & LSTM & Random Forest & Decision Tree \\
\hline Accuracy & $99.87 \%$ & $99.92 \%$ & $99.70 \%$ & $99.782 \%$ \\
\hline Logloss & 0.04384 & 0.00196 & 0.0749 & 0.0695 \\
\hline Time & $\sim 30 \mathrm{~min}$ & $\sim 90 \mathrm{~min}$ & $\sim 15 \mathrm{~min}$ & $\sim 18 \mathrm{~min}$ \\
\hline
\end{tabular}

Table III: Comparison of accuracy, logloss, and processing time between DNN and NON-DNNN neural networks in binary classification.

In this case, the lowest Logloss value was obtained using LSTM, with a value of 0.00196 . Table IV presents the results obtained in the confusion matrix for this system, comparing the actual class consisting of the test class used and the predicted class. We are observing the results of the percentage of values correctly predicted by the algorithm with an accuracy value higher than $99.9 \%$.

\begin{tabular}{|c|c|c|c|c|}
\hline \multirow{2}{*}{ True Class/Predicted Class } & \multicolumn{2}{|c|}{} & & \\
\cline { 2 - 5 } & Blacklist Flow & Anomaly-Spam Flow & $\begin{array}{c}\text { Anomaly-Sshscan } \\
\text { Flow }\end{array}$ & $\begin{array}{c}\text { Background Traffic } \\
\text { Data }\end{array}$ \\
\hline Blacklist Flow & 358 & 7 & 4 & 133319 \\
\hline Anomaly-Spam Flow & 0 & 140379 & 0 & 7529 \\
\hline Anomaly-Sshscan Flow & 0 & 0 & 108500 & 1424 \\
\hline Background Traffic Data & 2983 & 1983 & 30934 & 30520697 \\
\hline
\end{tabular}

Table IV: Results of The LSTM algorithm studied in this paper provided excellent results in terms of accuracy, with predicted values above $99.9 \%$, presenting high performance and a Logloss close to the target of 0.

The prediction is performed with a dataset containing a Background Traffic Data type flow corresponding to $99.8 \%$ of the data. A training dataset with real traffic could be a problem due to a large amount of traffic that a DNN type network must handle. On the other hand, the main problem detected in the training time used by LSTM being approximately nine times longer than that offered by MLP, RF, or DT algorithms.

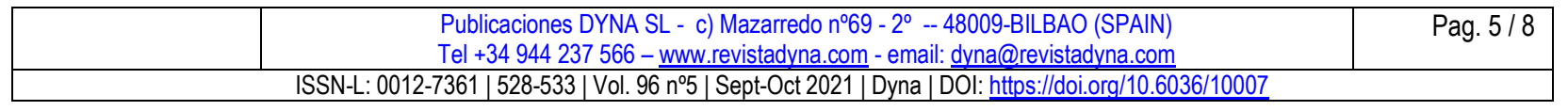




\begin{tabular}{|c|l|r|}
\hline & COMPARATIVE ANALYSIS AND EVALUATION OF THE APPLICATION OF & \\
Ingeniería e Industria & & \\
& & BUILDING \\
\hline RESEARCH ARTICLE & Xavier A. Larriva-Novo, Mario Vega-Barbas, Víctor A. Villagrá, Julio Berrocal & TECHNOLOGY \\
\hline
\end{tabular}

\subsection{Multiclass Clasification}

Multiclass classification has been used to predict each of the attack types presented in Table V. This experimentation, while maintaining the DNN system configuration used for the binary classification, employed three output nodes in the MLP and LSTM algorithms. Thus, each output node corresponded to a specific attack, except for the anomaly ssh-scan attack, which was not taken into account since it had a distribution close to 0 in the dataset.

In this case, the accuracy values changed compared to those obtained in the binary classification experiment. The RF accuracy increased by $0.08 \%$, and the corresponding DT accuracy decreased by $0.022 \%$. Logloss values for the non-DNN algorithms remained unchanged. On the other hand, DNN algorithms obtained mixed results. MLP remained unchanged in both accuracy and Logloss, but LSTM decreased its accuracy by $0.02 \%$ while achieving a lower Logloss. Consequently, it is observed that DNN type algorithms provide better performance for class classification

\begin{tabular}{|l|l|l|l|l|}
\hline \multirow{2}{*}{} & \multicolumn{3}{|l|}{ Deep Neural Network } & Non-Deep Neural Network \\
\cline { 2 - 5 } & MLP & LSTM & Random Forest & Decision Tree \\
\hline Accuracy & $99.87 \%$ & $99.9 \%$ & $99.78 \%$ & $99.76 \%$ \\
\hline Logloss & 0.04384 & 0.0013 & 0.0749 & 0.0695 \\
\hline Tiempo & $\sim 30 \mathrm{~min}$ & $\sim 90 \mathrm{~min}$ & $\sim 21 \mathrm{~min}$ & $\sim 28 \mathrm{~min}$ \\
\hline Blacklist Flow & 99.2 & 99.35 & 99.1 & 98.87 \\
\hline Anomaly-Sshscan Flow & 97.3 & 98.24 & 97.2 & 97.16 \\
\hline Anomaly-Spam Flow & 98.1 & 98.94 & 98.9 & 98.98 \\
\hline
\end{tabular}

Table V: Comparison of accuracy, Classes (attacks), and processing time between deep and non-deep neural networks in multiclass classification

In addition to the comparison of the two algorithms, this work provides an improvement in the learning time management of the algorithm. The time used to train the MLP algorithm was $30 \%$ longer with the use of the GPU than the time taken to train with the CPU. In turn, training the LSTM with the GPU spent about $75 \%$ less than that used by a CPU. The individual results for each of the algorithms used, in terms of CPU computation time, can be found in Table V. In addition, Table VI shows the results compared with similar works based on no-deep learning algorithms. Here we can see that our model based on a LSTM network achieves an accuracy of $99 \%$ with a modern dataset such as UGR16

\begin{tabular}{|l|l|l|l|l|l|l|}
\hline & Algoritmo & Accuracy BC & LogLoss MC & DATASET & Presision & Recall \\
\hline Proposed Model & LSTM & 99.92 & 0.0013 & UGR16 & 99.11 & 96.57 \\
\hline$[16]$ & LSTM & 97.3 & $\mathrm{X}$ & KDD99 & $\mathrm{x}$ & $\mathrm{x}$ \\
\hline$[18]$ & LSTM & 93.82 & $\mathrm{X}$ & KDD99 & $\mathrm{x}$ & $\mathrm{x}$ \\
\hline$[19]$ & MLP & 91 & $\mathrm{X}$ & DARPA & $\mathrm{x}$ & $\mathrm{x}$ \\
\hline$[6]$ & MLP & 95.87 & $\mathrm{X}$ & KDD99 & $\mathrm{x}$ & $\mathrm{x}$ \\
\hline
\end{tabular}

Table VI: Comparison of related works and the proposed model

\section{CONCLUTIONS}

Throughout this article, the authors have shown an analysis of the accuracy, performance, and Logloss of different machine learning methods for the classification of real traffic and the identification of possible attacks. In particular, two possible approaches based on deep machine learning algorithms and architectures such as LSTM and MLP have been presented. These results have been contrasted with models traditionally used at the level of intrusion detection systems individually [2] and which are developed based on traditional machine learning techniques (non-DNN). Decision trees, like the random forest model, provide high accuracy. However, new deep learning models are emerging that, despite their difficulty of elaboration, have generated high performance in terms of accuracy. The use of current deep learning algorithms is becoming easier and easier since the large companies that compete in their development are offering extensive development frameworks on them [32].

This study also presents the analysis of the learning times for each development for CPU and GPU-based execution environments. According to the results presented, the LSTM type DNN algorithm for the developed binary classification model has obtained the best accuracy and Logloss results, with values of $99.92 \%$ and 0.00196 , respectively. In addition, we can identify that the values corresponding to the attacks detected incorrectly represent $0.067 \%$ of the total data flows. This allows us to consider that this is an acceptable value in terms of accuracy for other studies analyzed. In addition, this allows us to meet one of the objectives proposed by the study, which is to increase the accuracy of the proposed models, as shown in Table VI. Similarly, in class classification, the results are equally accurate, with the difference that here not only is it detected if the traffic is anomalous, but also the type of attack that is 


\begin{tabular}{|c|l|r|}
\hline & COMPARATIVE ANALYSIS AND EVALUATION OF THE APPLICATION OF & \\
Ingeniería e Industria & & \\
& & BUILDING \\
\hline RESEARCH ARTICLE & Xavier A. Larriva-Novo, Mario Vega-Barbas, Víctor A. Villagrá, Julio Berrocal & TECHNOLOGY \\
\hline
\end{tabular}

occurring, with an accuracy of $99.9 \%$ and a Logloss of 0.0013 , in this case, the LogLoss value is lower than that of the binary classification, approaching the reference value of 0 , although the accuracy of both models is very close with a variation of $0.03 \%$. The LogLoss is lower in the latter.

Concerning the datasets, different researchers have used the KDD collection to obtain better results for IDSs based on anomalies. For this research, we chose to use the UGR16, obtaining a high accuracy value and small Logloss values. The proposed LSTM-type DNN algorithm gives us the best performance results compared to other models described in Section 2 and to some non-DNN neural network algorithms tested in this study. In addition, this research provides an evaluation of such a dataset with deep learning tools, an aspect not addressed to date [2], [24].

Another conclusion drawn from this research work is the current problem of handling large quantities of datasets for processing. The authors intend to use in future research the algorithms applied to the dataset treated in this article to new ones to define additional configuration parameters. Furthermore, it is planned to explore additional data normalization models.

The use of realistic datasets facilitates the development of more efficient and effective intrusion or attack detection systems. However, for a dataset to be true to reality, its size, the amount of information it contains must grow gradually. In this sense, the in-depth study of the UGR16 dataset for its application to the context of cybersecurity is an important work to be carried out since it facilitates the application of the models generated under its protection to real contexts.

Additionally, this study shows a clear difference in the implementation of deep learning type algorithms with those presented in related works, as well as the contribution of LogLoss metrics, precision and recall to identify the accuracy of the model classification. This allows extending future research to analyze new IDS models based on anomalies that apply other types of deep learning algorithms, based on the various parameters proposed here, the use of new and more efficient configurations, as well as new methods such as leave-one-out cross-validation.

\section{REFERENCES}

[1] V. Chandola, A. Banerjee, and V. Kumar, “Anomaly detection: A survey," ACM Computing Surveys, vol. 41, no. 3, pp. 1-58, Jul. 2009, doi: 10.1145/1541880.1541882.

[2] A. Aldweesh, A. Derhab, and A. Z. Emam, "Deep learning approaches for anomaly-based intrusion detection systems: A survey, taxonomy, and open issues," Knowledge-Based Systems, vol. 189, p. 105124, Feb. 2020, doi: 10.1016/j.knosys.2019.105124.

[3] G. C. Tjhai, M. Papadaki, S. M. Furnell, and N. L. Clarke, "Investigating the problem of IDS false alarms: An experimental study using Snort," in Proceedings of The Ifip Tc 11 23rd International Information Security Conference, vol. 278, S. Jajodia, P. Samarati, and S. Cimato, Eds. Boston, MA: Springer US, 2008, pp. $253-267$.

[4] L. Hu, T. Li, N. Xie, and J. Hu, "False positive elimination in intrusion detection based on clustering," in 201512 th International Conference on Fuzzy Systems and Knowledge Discovery (FSKD), Aug. 2015, pp. 519-523, doi: 10.1109/FSKD.2015.7381996.

[5] S. Hochreiter and J. Schmidhuber, "Long Short-Term Memory," Neural Computation, vol. 9, no. 8, pp. 1735-1780, Nov. 1997, doi: 10.1162/neco.1997.9.8.1735.

[6] P. Barapatre, N. Z. Tarapore, S. G. Pukale, and M. L. Dhore, "Training MLP neural network to reduce false alerts in IDS," in 2008 International Conference on Computing, Communication and Networking, Dec. 2008, pp. 1-7, doi: 10.1109/ICCCNET.2008.4787714.

[7] M. Tavallaee, E. Bagheri, W. Lu, and A. A. Ghorbani, "A detailed analysis of the KDD CUP 99 data set," in 2009 IEEE Symposium on Computational Intelligence for Security and Defense Applications, Jul. 2009, pp. 1-6, doi: 10.1109/CISDA.2009.5356528.

[8] G. Creech and J. Hu, "Generation of a new IDS test dataset: Time to retire the KDD collection," in 2013 IEEE Wireless Communications and Networking Conference (WCNC), Apr. 2013, pp. 4487-4492, doi: 10.1109/WCNC.2013.6555301.

[9] G. Maciá-Fernández, J. Camacho, R. Magán-Carrión, P. García-Teodoro, and R. Theron, "UGR '16: A new dataset for the evaluation of cyclostationarity-based network IDSs," Computers \& Security, vol. 73, pp. 411-424, 2018.

[10] A. Liaw and M. Wiener, "Classification and Regression by randomForest," vol. 2, p. 6, 2002.

[11] M. A. Friedl and C. E. Brodley, "Decision tree classification of land cover from remotely sensed data," Remote Sensing of Environment, vol. 61, no. 3, pp. 399-409, Sep. 1997, doi: 10.1016/S0034-4257(97)00049-7.

[12] R. Caruana and A. Niculescu-Mizil, “An empirical comparison of supervised learning algorithms,” 2006, pp. 161168, doi: 10.1145/1143844.1143865.

[13] R. Bali, D. Sarkar, and T. Sharma, Learning Social Media Analytics with R. Packt Publishing Ltd, 2017.

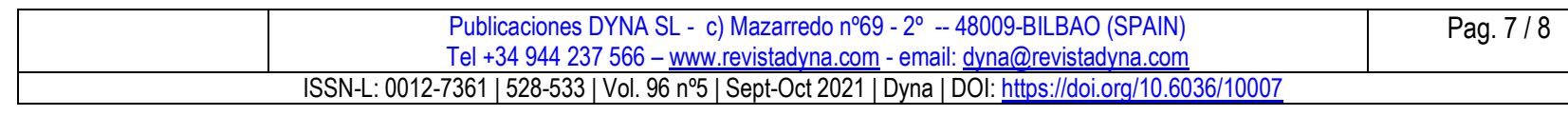




\begin{tabular}{|c|l|r|}
\hline & COMPARATIVE ANALYSIS AND EVALUATION OF THE APPLICATION OF \\
Ingeniería e Industria & & \\
& & BUILDING \\
\hline RESEARCH ARTICLE & Xavier A. Larriva-Novo, Mario Vega-Barbas, Víctor A. Villagrá, Julio Berrocal & TECHNOLOGY \\
\hline
\end{tabular}

[14] L. Deng, "Deep Learning: Methods and Applications," Foundations and Trends® in Signal Processing, vol. 7, no. 3-4, pp. 197-387, 2014, doi: 10.1561/2000000039.

[15] W. Wang et al., "HAST-IDS: Learning Hierarchical Spatial-Temporal Features Using Deep Neural Networks to Improve Intrusion Detection," IEEE Access, vol. 6, pp. 1792-1806, 2018, doi: 10.1109/ACCESS.2017.2780250.

[16] G. Kim, H. Yi, J. Lee, Y. Paek, and S. Yoon, "LSTM-Based System-Call Language Modeling and Robust Ensemble Method for Designing Host-Based Intrusion Detection Systems," arXiv:1611.01726 [cs], Nov. 2016, Accessed: May 22, 2018. [Online]. Available: http://arxiv.org/abs/1611.01726.

[17] X. Larriva-Novo, C. Sánchez-Zas, V. A. Villagrá, M. Vega-Barbas, and D. Rivera, "An Approach for the Application of a Dynamic Multi-Class Classifier for Network Intrusion Detection Systems,” Electronics, vol. 9, no. 11, Art. no. 11, Nov. 2020, doi: 10.3390/electronics9111759.

[18] Z. Chiba, N. Abghour, K. Moussaid, A. El Omri, and M. Rida, "New anomaly network intrusion detection system in cloud environment based on optimized back propagation neural network using improved genetic algorithm," International Journal of Communication Networks and Information Security, vol. 11, no. 1, pp. 61-84, 2019.

[19] D. Kwon, H. Kim, J. Kim, S. C. Suh, I. Kim, and K. J. Kim, “A survey of deep learning-based network anomaly detection," Cluster Computing, Sep. 2017, doi: 10.1007/s10586-017-1117-8.

[20] L. F. Maimó, F. J. G. Clemente, M. G. Pérez, and G. M. Pérez, "On the performance of a deep learning-based anomaly detection system for 5G networks,” in 2017 IEEE SmartWorld, Ubiquitous Intelligence \& Computing, Advanced \& Trusted Computed, Scalable Computing \& Communications, Cloud \& Big Data Computing, Internet of People and Smart City Innovation (SmartWorld/SCALCOM/UIC/ATC/CBDCom/IOP/SCI), 2017, pp. 1-8.

[21] M. Moradi and M. Zulkernine, "A neural network based system for intrusion detection and classification of attacks," in Proceedings of the IEEE International Conference on Advances in Intelligent Systems-Theory and Applications, 2004, pp. 15-18.

[22] “MIT Lincoln Laboratory: DARPA Intrusion Detection Evaluation.” https://www.ll.mit.edu/ideval/data/ (accessed May 22, 2018).

[23] X. A. Larriva-Novo, M. Vega-Barbas, V. A. Villagra, and M. Sanz Rodrigo, "Evaluation of Cybersecurity Data Set Characteristics for Their Applicability to Neural Networks Algorithms Detecting Cybersecurity Anomalies," IEEE Access, vol. 8, pp. 9005-9014, 2020, doi: 10.1109/ACCESS.2019.2963407.

[24] P. Mishra, V. Varadharajan, U. Tupakula, and E. S. Pilli, “A Detailed Investigation and Analysis of using Machine Learning Techniques for Intrusion Detection,” IEEE Communications Surveys Tutorials, pp. 1-1, 2018, doi: 10.1109/COMST.2018.2847722.

[25] D. Wu, Z. Jiang, X. Xie, X. Wei, W. Yu, and R. Li, "LSTM learning with Bayesian and Gaussian processing for anomaly detection in industrial IoT," IEEE Transactions on Industrial Informatics, vol. 16, no. 8, pp. 5244-5253, 2019.

[26] S. Cai, "Predicting rental listing popularity : 2 Sigma connect Renthop," Thesis, 2017.

[27] “Keras Documentation.” https://keras.io/ (accessed Jun. 04, 2018).

[28] "TensorFlow," TensorFlow. https://www.tensorflow.org/?hl=es (accessed Jan. 14, 2019).

[29] M. Cassel and F. Lima, "Evaluating one-hot encoding finite state machines for SEU reliability in SRAM-based FPGAs," in 12th IEEE International On-Line Testing Symposium (IOLTS'06), Jul. 2006, p. 6 pp.-, doi: 10.1109/IOLTS.2006.32.

[30] "sklearn.preprocessing.LabelEncoder _ $\quad$ scikit-learn $\quad 0.24 .1 \quad$ documentation." https://scikitlearn.org/stable/modules/generated/sklearn.preprocessing.LabelEncoder.html (accessed Mar. 30, 2021).

[31] X. Larriva-Novo, M. Vega-Barbas, V. A. Villagrá, D. Rivera, M. Álvarez-Campana, and J. Berrocal, "Efficient Distributed Preprocessing Model for Machine Learning-Based Anomaly Detection over Large-Scale Cybersecurity Datasets," Applied Sciences, vol. 10, no. 10, Art. no. 10, Jan. 2020, doi: 10.3390/app10103430.

[32] A. Géron, Hands-On Machine Learning with Scikit-Learn, Keras, and TensorFlow: Concepts, Tools, and Techniques to Build Intelligent Systems. O’Reilly Media, 2019.

\section{AGRADECIMIENTOS}

The research presented in this article has been partially funded by the PLICA project within the COINCIDENTE program of the Ministerio de Defensa de España.

\section{SUPPLEMENTARY MATERIAL}

https://www.revistadyna.com/documentos/pdfs/_adic/10007-1.pdf

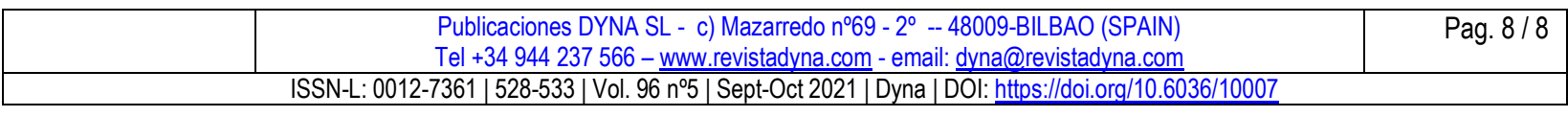

